\title{
Climate sensitivity across marine domains of life: limits to evolutionary adaptation shape species interactions
}

\author{
DANIELA STORCH ${ }^{1}$, LENA MENZEL ${ }^{1}$, STEPHAN FRICKENHAUS ${ }^{2}$ and HANS-O. PÖRTNER ${ }^{1}$ \\ ${ }^{1}$ Department of Integrative Ecophysiology, Alfred-Wegener-Institute Helmholtz Center for Polar- and Marine Research, \\ Bremerhaven 27570, Germany, ${ }^{2}$ Department of Scientific Computing, Alfred-Wegener-Institute Helmholtz Center for Polar-and \\ Marine Research, Bremerhaven 27570, Germany
}

\begin{abstract}
Organisms in all domains, Archaea, Bacteria, and Eukarya will respond to climate change with differential vulnerabilities resulting in shifts in species distribution, coexistence, and interactions. The identification of unifying principles of organism functioning across all domains would facilitate a cause and effect understanding of such changes and their implications for ecosystem shifts. For example, the functional specialization of all organisms in limited temperature ranges leads us to ask for unifying functional reasons. Organisms also specialize in either anoxic or various oxygen ranges, with animals and plants depending on high oxygen levels. Here, we identify thermal ranges, heat limits of growth, and critically low (hypoxic) oxygen concentrations as proxies of tolerance in a meta-analysis of data available for marine organisms, with special reference to domain-specific limits. For an explanation of the patterns and differences observed, we define and quantify a proxy for organismic complexity across species from all domains. Rising complexity causes heat (and hypoxia) tolerances to decrease from Archaea to Bacteria to uni- and then multicellular Eukarya. Within and across domains, taxon-specific tolerance limits likely reflect ultimate evolutionary limits of its species to acclimatization and adaptation. We hypothesize that rising taxon-specific complexities in structure and function constrain organisms to narrower environmental ranges. Low complexity as in Archaea and some Bacteria provide life options in extreme environments. In the warmest oceans, temperature maxima reach and will surpass the permanent limits to the existence of multicellular animals, plants and unicellular phytoplankter. Smaller, less complex unicellular Eukarya, Bacteria, and Archaea will thus benefit and predominate even more in a future, warmer, and hypoxic ocean.
\end{abstract}

Keywords: adaptation, Archaea, Bacteria, complexity of organisms, domains of life, Eukarya, evolution, hypoxia tolerance, oxygen and capacity limited thermal tolerance, thermal tolerance

Received 30 January 2014; revised version received 15 May 2014 and accepted 15 May 2014

\section{Introduction}

Marine organisms from all domains experience climate change and their ecosystems respond by altered diversity, function and community dynamics (Karl et al., 2001; Parmesan \& Matthews, 2005; Hoegh-Guldberg \& Bruno, 2010). Marine organisms are directly affected through increasing seawater temperatures in most habitats and, concomitantly, by decreasing ambient oxygen concentrations due to warming. Deep ocean temperature presently is around $2{ }^{\circ} \mathrm{C}$ (Mantyla \& Reid, 1983) and the average global sea surface temperature (SST) is $17^{\circ} \mathrm{C}$. Typically, the world's SST ranges from $-2{ }^{\circ} \mathrm{C}$ in polar regions (Gilmour, 1979) up to hot summer maxima of $41{ }^{\circ} \mathrm{C}$ in tropical regions (McKenzie, 1994; McKenzie \& Campbell, 2004); extreme water

Correspondence: Daniela Storch, tel. +49 4714831 1934, fax +49 4714831 1149, e-mail: Daniela.Storch@awi.de; Hans-O. Pörtner, tel. +49 4714831 1307, fax +49 4714831 1149,

e-mail: Hans.Poertner@awi.de temperatures around hydrothermal vents at the sea floor reach about $400{ }^{\circ} \mathrm{C}$ (Haase et al., 2007) and are far beyond the limits to life. Future global surface temperatures (ocean and land) are projected to increase by up to $4.5^{\circ} \mathrm{C}$ above pre-industrial values by 2100 , and by up to $8.5^{\circ} \mathrm{C}$ by 2300 (Meinshausen et al., 2011), driven by $\mathrm{CO}_{2}$ emissions following representative concentration pathway 8.5 (Moss et al., 2008).

Warming trends and regional freshening due to icemelt and enhanced precipitation increase density stratification of marine waters and reduce mixing between surface and deeper water layers. As a consequence, the transfer of oxygen $\left(\mathrm{O}_{2}\right)$ from the well-oxygenated surface to mid-water layers becomes limiting. At the same time, oxygen in mid-water layers is depleted by microbial and animal respiration as these organisms consume the organic material formed by photosynthetic algae close to the surface and sinking down deep over time. $\mathrm{O}_{2}$ concentrations in marine waters presently range from hyperoxic levels of $1500 \mu \mathrm{M} \mathrm{O}_{2}$ in illuminated surface waters with high 
photosynthetic activity (Irwin \& Davenport, 2002) to levels in mid-water layers which can reach below $60 \mu \mathrm{O}_{2}$ (termed hypoxia) and even fall below 4-5 $\mathrm{M} \mathrm{O}_{2}$ (termed suboxia) (Deutsch et al., 2011). The oxygen content of surface water saturated with air ranges between $367 \mu \mathrm{M} \mathrm{O} \mathrm{O}_{2}$ at $0{ }^{\circ} \mathrm{C}$ and $170 \mu \mathrm{M} \mathrm{O}_{2}$ at $40{ }^{\circ} \mathrm{C}$, at a mean salinity of 32 PSU (Practical Salinity Units) and an atmospheric pressure of $1 \mathrm{~atm}$ (see also Figure S1). In warming hyper-saline seawater equilibrated with air, $\mathrm{O}_{2}$ levels can fall below the threshold of $60 \mu \mathrm{M} \mathrm{O} \mathrm{O}_{2}$ commonly assumed to elicit transition to hypoxia-adapted ecosystems (Figure S1). Ambient temperature, oxygen availability to organisms, and their oxygen demand are thus closely intertwined.

Increased density-induced stratification and/or the warming-induced rise in $\mathrm{O}_{2}$ demand in sediments and mid-water layers presently cause an expansion of hypoxic, suboxic, and anoxic areas, exacerbated by coastal eutrophication which enhances the biomass and $\mathrm{O}_{2}$ demand of heterotrophic organisms. The number of coastal anoxic 'dead' zones void of metazoan life has increased dramatically since 1960 (Díaz \& Rosenberg, 2008). By 2100, the $\mathrm{O}_{2}$ content of the oceans is projected to fall by $1-7 \%$ (Keeling et al., 2010). Future changes involve a solubility effect as SST increments by 0.2 $3.8{ }^{\circ} \mathrm{C}$ (Bopp et al., 2013) would cause a decrease in $\mathrm{O}_{2}$ solubility by $0.5-9.0 \%$ in cold seawater $\left(0{ }^{\circ} \mathrm{C}\right)$ and by $0.3-5.2 \%$ in warm tropical seawater $\left(40{ }^{\circ} \mathrm{C}\right.$ ) (Figure S1; Data S6). Low $\mathrm{O}_{2}$ conditions are stressful for aerobic organisms and, depending on the level of hypoxia reached (Figure S1), exclude sensitive species and favor hypoxia tolerant species or facultative or obligate anaerobes. It should be noted that constraints in oxygen supply to animals may set in early in some species (e.g., Fiala-Médioni, 1979; Naylor et al., 1999), even slightly below air saturation.

Developing a cause and effect understanding for ecosystem change requires identifying the unifying principles of organism responses to relevant drivers such as temperature and oxygen and their implications for species interactions in organisms from all domains. Temperature-dependent performance curves (thermal reaction norms), for example, indicate the functional optimization and specialization of species in limited temperature ranges (Deutsch et al., 2008). Differences in the positioning of these reaction norms on the temperature scale define the temperature range and area where species coexist and interact, their relative performance and thereby, their competitive success (Pörtner \& Farrell, 2008).

In search of a wider concept that makes such principles understood for organisms across all domains of marine life, we built on the concept of oxygen and capacity limited thermal tolerance (OCLTT) developed for animals (Pörtner, 2002). We used this concept as an analogue and adopted the view that organismic complexity sets the highest possible thermal (and other) tolerance limits (e.g., hypoxia). In animals, for example, limits are first seen at the highest level of functional complexity such as cardio-circulation and its capacity to sustain oxygen supply. At more extreme temperatures beyond these limits, rates of key functions such as growth or activity are falling (Pörtner \& Knust, 2007; Storch et al., 2011). While animals experience constraints in oxygen supply to tissues and associated energy budget, the equivalent constraint in unicellular eukaryotes may be the coordination between cytosol and organelles and in Archaea and Bacteria presumably the coordination of non-organellar cellular compartments being limited by the stability and integrity of membrane-molecular complexes (Daniel et al., 2004).

From this reasoning, complexity levels might relate to the levels of tolerance under exposure to the respective extremes of temperature and/or hypoxia. The Eukarya, for the sake of being more complex and larger, may be less tolerant than the less complex and much smaller Archaea and Bacteria, and the multicellular Eukarya less so than the unicellular ones. Here, we introduce a simple complexity indicator of organisms, which enables us to correlate complexity to maximal thermal limits of organisms. To test its applicability, we performed a meta-analysis of published literature data and examined the ranges of tolerance to various levels of temperatures and dissolved oxygen within and across organism domains. We assessed the upper thermal limits for growth and analyzed critical oxygen concentrations as a proxy for oxygen requirements of organisms. Then, we interpret our findings in light of the proposed framework of differential complexity to identify the reasons for different sensitivities across domains in the context of climate change.

\section{Material and methods}

\section{Classification of domains and groups}

We used the natural system of organisms divided into the three domains Archaea, Bacteria, and Eukarya, and grouped individual taxa, verified after Woese and co-authors (Woese et al., 1990). The domain 'Eukarya' was subdivided into less complex unicellular and more complex multicellular organisms. We excluded the group of 'green non-sulfur Bacteria' as we could not find any report on marine species. Endoparasitic organisms, e.g., endoparasitic Bacteria, and all eukaryotic Microsporidia were not considered, as they depend on the habitat preferences of their hosts. We grouped the unicellular Eukarya according to Woese et al. (1990) together with the 
important marine group of microalgae. Species were considered marine when they were collected from marine habitats, and hydrothermal, if collected from marine hydrothermal habitats, opposed to non-hydrothermal habitats (sum of benthic and pelagic). To avoid duplicating data reported repeatedly for the same species, we focused on 'accepted marine species' in the World Register of Marine Species (Costello et al., 2013; WoRMS, 01.11.2012, all domains). We had their names validated in the 'List of Prokaryotic names with Standing in Nomenclatures' (LPSN, 30.11.2012) (Bacteria and Archaea). Cyanobacteria are excluded from the LPSN database and were not considered in this data set.

\section{Organismic complexity}

For the species growing at the highest temperature in each of 14 groups, organismic complexity was quantified as the sum of functional compartments at all levels of biological organization (Fig. 1; Table S1). For multicellular Eukarya, we counted organ systems and also counted the differentiation of 'organs', 'tissues' and cell types into various functions. At the lowest complexity levels, we identified the functional compartmentation of a single cell, counting functionally different non-organellar and organellar cell compartments.

\section{Meta-analysis 'thermal tolerances'}

Upper and lower temperature limits for growth (Tmax and Tmin) of marine eukaryotic species were compiled for each group building on a Google Scholar search using the keywords 'maximal temperature' or 'Tmax', 'temperature' 'growth', 'group name', 'marine', and their combinations. For Bacteria (Data S3) and Archaea (Data S4), data were extracted from the original species descriptions. If only the temperature of no growth was given instead of a minimum and maximum temperature, positive growth was assumed to span the temperature range from the given minimum temperature of no growth $+1{ }^{\circ} \mathrm{C}$ to the given maximum temperature of no growth $-1{ }^{\circ} \mathrm{C}$. For all domains, differences between Tmax values of hydrothermal and non-hydrothermal organisms (nonhydrothermal is defined as the sum of benthic and pelagic) were evaluated from Data S1-S4. To avoid duplicating information on growth in species studied more than once, the study with the highest detected Tmax value was chosen; if Tmax values were identical in both studies then the study reporting the lowest value of Tmin was selected. Species named as 'spec.' were assumed to be different within and between studies. The highest detected temperature of positive growth in a species or taxon was taken as the upper temperature limit for growth of the corresponding taxon. In addition, we identified the differences in thermal tolerance and in thermal ranges between domains from the temperature range of growth $(\Delta \mathrm{T}=\mathrm{Tmax}-\mathrm{Tmin})$ of each species.

\section{Meta-analysis 'oxygen tolerances'}

The oxygen range supporting marine life at different complexity levels was approached differently for the three domains. Bacteria and Archaea were classified according to their metabolic patterns as aerobic (grouping both strictly aerobic and aerobic) and anaerobic (grouping facultatively anaerobic, anaerobic, and strictly anaerobic). For Eukarya, we identified

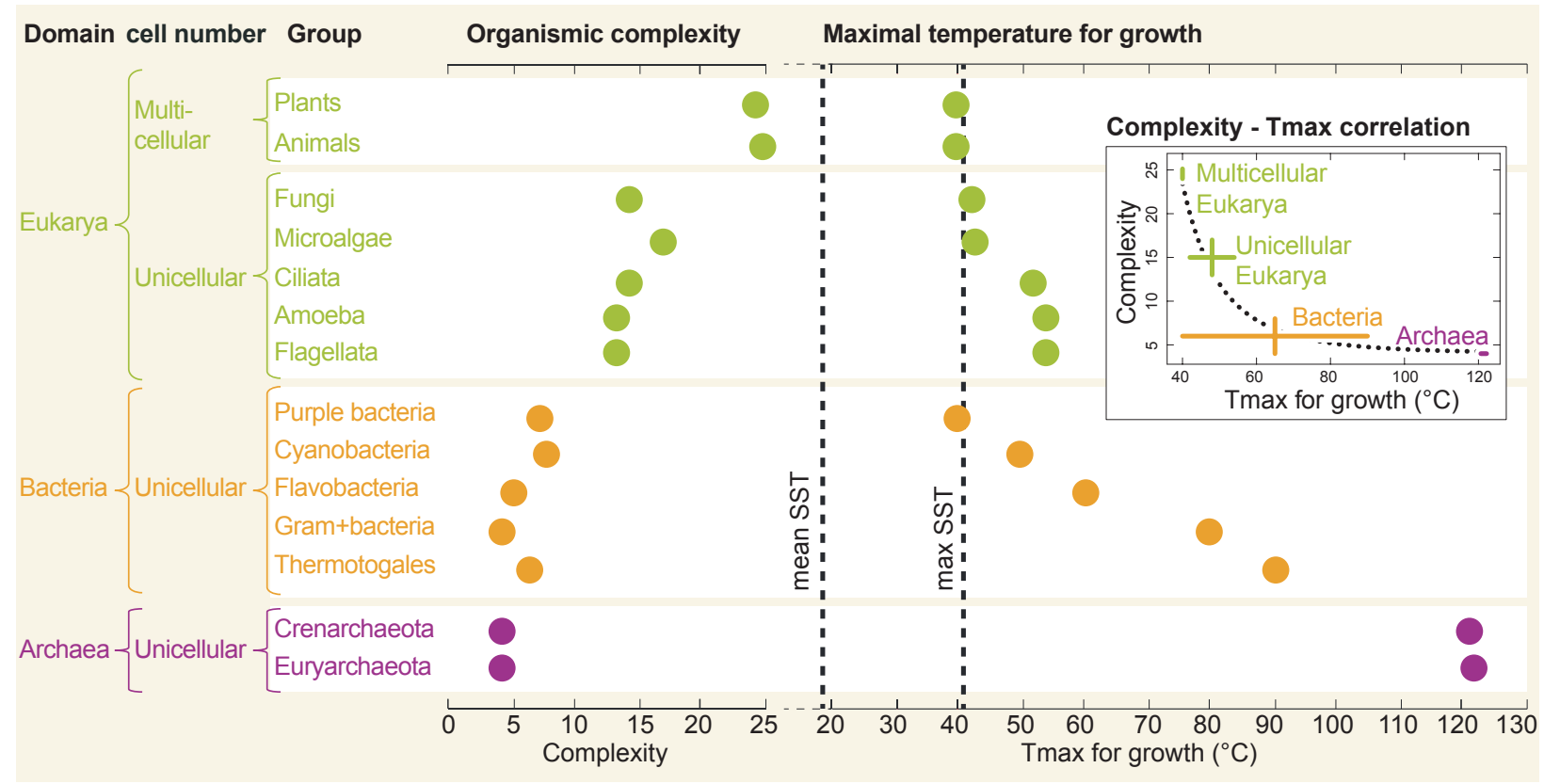

Fig. 1 Organismic complexity at maximal thermal limits within domains and groups (sensu Woese et al., 1990). Organismic complexity was exemplified for the species with the highest Tmax for growth within each group (Table S1). With increasing complexity, thermal tolerance is found to decrease (Inset). High organismic complexity enables an increase in body size, associated with decreasing heat tolerance. Inset: fit $=\left(84{ }^{\circ} \mathrm{C} / \mathrm{Tmax}\right)^{4}+4$ (Figure S1; Data S6)., where Tmax, maximal temperature $\left({ }^{\circ} \mathrm{C}\right)$; SST, sea surface temperature. 
the critical oxygen concentration $\left(\mathrm{O}_{2}\right.$ crit) (Data S5), we searched Google Scholar for the keywords 'critical oxygen tension $\left(\mathrm{P}_{\mathrm{O} 2 \text { crit }}\right)$ ' and 'marine' in combination with 'group/phyla names'. We only considered publications reporting data on temperature, salinity, and body weight of the organism. If more than one report was found for the same species, we chose the publication reporting the lowest oxygen thresholds. $\mathrm{P}_{\mathrm{O} 2 \text { crit }}$ values were converted to critical oxygen concentrations $\left(\mathrm{O}_{2}\right.$ crit) considering oxygen solubility in sea-water depending on salinity (adopting 32 PSU as the salinity mean from all reports $\geq 30$ PSU) and reported temperatures. Dry weight was converted to wet weight. We grouped Eukarya into small and large according to their body weight using different threshold weights.

\section{Statistics}

The equation describing the correlation of complexity with Tmax was determined with the program ' $R$ ' (Figure S1; Data S6). Within each domain, the range of Tmax for growth was plotted vs. the range of organismic complexity levels. The correlation curve was fitted empirically. A one-sided Wilcoxon rank sum test (Wilcoxon, 1945) was applied to test the values of Tmax for differences between Archaea, Bacteria, or Eukarya taking into account habitat (hydrothermal, nonhydrothermal) according to data availability. For testing whether the widths of thermal windows are significantly different between Archaea, Bacteria or Eukarya, we performed a two-sided Wilcoxon rank sum test using the BenjaminiHochberg correction of $P$-values for multiple testing (Benjamini \& Hochberg, 1995). Within defined oxygen concentration ranges, we tested whether small multicellular Eukarya are more tolerant to hypoxia than large ones. The class boundary (separating 'small' from 'large') was sampled uniformly resulting in 30 different evaluations (corresponding to an interval of six standard errors of means around the median of body weight). Error bars in the accumulated bins of the histogram reflect the standard deviation over the 30 comparisons. For all statistical tests, the program ' $R$ ' was used (Figure S1; Data S6).

\section{Results and discussion}

We identified the multicellular Eukarya as the most complex organisms, followed by the unicellular Eukarya and Bacteria and the Archaea as the simplest (Table S1). The function at the highest organizational level in multicellular Eukarya would be the blood circulation tied to the circulatory system, which serves as a supply and hormonal communication system between organs in animals. In unicellular Eukarya, the function identified at the highest level would be the coordination between the cytosolic and cellular sub-compartments (especially organelles). So-called microcompartments in Bacteria and Archaea are nonorganellar such that separation may be incomplete facilitating communication. Most microcompartments do not contain lipids as they are not surrounded by membranes but protein shells which usually enclose various enzymes (Table S1). While animals at upper thermal limits experience constraints in oxygen supply capacity and cost to tissues (OCLTT), the equivalent in unicellular Eukarya might be the capacity and costs of solute transport across compartmental membranes. In Archaea and Bacteria, it might be the solute transport across compartmental borders being limited by the integrity of macromolecular complexes (Daniel et al., 2004). From a different angle, such integrity might reflect a rate controlling system limiting tolerance (Corkrey et al., 2012), e.g., at the level of molecular (including lipid protein) complexes in unicellular organisms and at the level of oxygen supply in animals. From modeling meta-data of temperature-dependent growth rates in Bacteria, Archaea, and unicellular Eukarya, Corkrey et al. suggest that thermodynamic properties of protein hydration govern biological rates of all unicellular poikilotherm life and that a single enzymecatalyzed reaction limits growth rate at all temperatures. However, this model does not address functions at the highest complexity level and thus does not explain the specific differences between temperature limits of various domains where membrane and protein adaptation follows the thermal ranges of the whole organism, up to $120^{\circ} \mathrm{C}$ in Archaea. The model does also not include functional limitations in multicellular organisms where earliest thermal limits are found at higher than molecular complexity levels (see above). Limits seen at molecular levels are usually more extreme than seen in the whole organism. Much wider than whole organism tolerance ranges have even been found in isolated mitochondria, which also include such macromolecular complexes and rely on their functioning. In light of the likely principle of a characteristic high-level function shaping the limits of all organisms in a domain, the hypothesis arises that the highest limits found among species of a taxon, in relation to its complexity level, would then be the maximum limit possible for any species of that taxon. That limit cannot be overcome by evolutionary adaptation unless a reduction in complexity and a shift towards a limiting function at lower complexity levels occur. On evolutionary time scales, such a drop in complexity has only been found at the community level when e.g., during the global reef collapse of the Late Devonian more complex groups disappeared first as the reef ecosystem collapsed (Copper, 1994). A reduction in complexity may also happen with the emergence of new species with different functional characteristics (Fig. 1).

Accordingly, organismic complexity may relate to thermal and hypoxia tolerances and also set the wider 
framework for interactions between species from different domains. The large, complex multicellular Eukarya would be less tolerant than the unicellular ones, and the Eukarya in general less tolerant than the less complex Archaea and Bacteria. We evaluated complexity levels and thermal maxima for species from 14 major groups (sensu Woese et al., 1990) of marine organisms from the three domains (Fig. 1; Table S1). Following the principles of the OCLTT concept, we did not identify lethal limits, but rather the ecologically more relevant upper temperature limits to positive growth ('Tmax'). Highest Tmax values, up to $122{ }^{\circ} \mathrm{C}$, are found among Archaea followed by Bacteria with thresholds up to $90{ }^{\circ} \mathrm{C}$, higher than the $54{ }^{\circ} \mathrm{C}$ limit in unicellular Eukarya, which exceeds the $40^{\circ} \mathrm{C}$ threshold in multicellular Eukarya. The decrease in thermal tolerance is correlated with the rising complexity of domains and taxa therein. Eukarya differentiate into uni- and multicellular organisms (Fig. 1, inset). Archaea and Bacteria also differentiate into taxa of various complexities but altogether, not only display higher thresholds (corrected $P<0.0001$ ) but also wider temperature ranges for growth than the Eukarya (Fig. 2; Data S1-S4; corrected $P<0.0001)$. Lower degrees of compartmentation may provide an advantage to hyperthermophiles among Archaea and Bacteria. In other Bacteria, the existence of intracellular microcompartments such as carboxysomes and chromatophores may set their limits to lower temperatures than in Archaea, which mostly lack microcompartments. High cellular complexity due to the use of both, mitochondria and chloroplasts by marine unicellular, eukaryotic algae may explain their even lower Tmax of $43^{\circ} \mathrm{C}$, lower than in heterotrophic unicellular Eukarya and comparable to the Tmax of multicellular Eukarya.

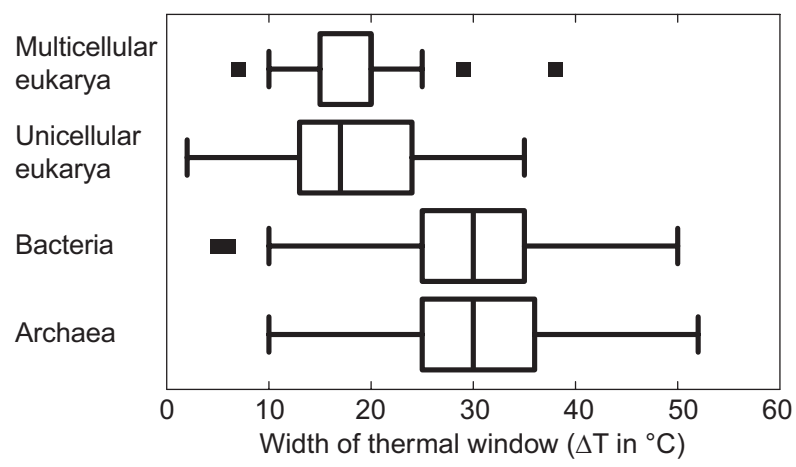

Fig. 2 Temperature ranges for positive growth $(\Delta \mathrm{T})$ of multiand unicellular Eukarya, Bacteria, and Archaea. Species numbers of Eukarya: multicellular $N=55$, unicellular $N=125$; Bacteria: $N=823$; Archaea: $N=77$. Box-and-whisker plots with outliers. $\Delta \mathrm{T}=\mathrm{Tmax}-\mathrm{Tmin}$ (Data S1-S4).
Among Archaea $(N=78)$, Bacteria $(N=856)$, and Eukarya (unicellular $N=165$; multicellular $N=53$ ), the highest thresholds within each domain were found in hydrothermal environments (Data S1-S4), which have extreme ambient temperatures beyond those set by climate and far beyond those tolerated by life. Tmax values found there may thus reflect the evolutionary limits of the domain to heat adaptation, which are higher in Archaea than in Bacteria and higher in both of them than in Eukarya. Interestingly, Archaea grow at warmer temperatures than Bacteria not only in hydrothermal $(P<0.0001)$ but even in non-hydrothermal $(P<0.0001)$ environments (Fig. 3$)$. The low numbers of hydrothermal Eukarya studied regarding their temperature-dependent growth preclude us from comparing hydrothermal and non-hydrothermal uni- and multicellular Eukarya.

In non-hydrothermal environments, almost half of the Archaea and Bacteria display Tmax values above $41{ }^{\circ} \mathrm{C}$, the maximal temperature of the open ocean, whereas only very few Eukarya can grow at these
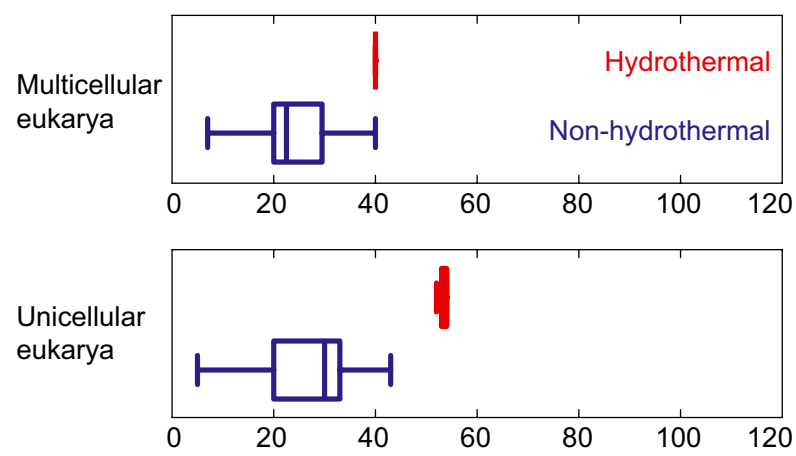

Bacteria
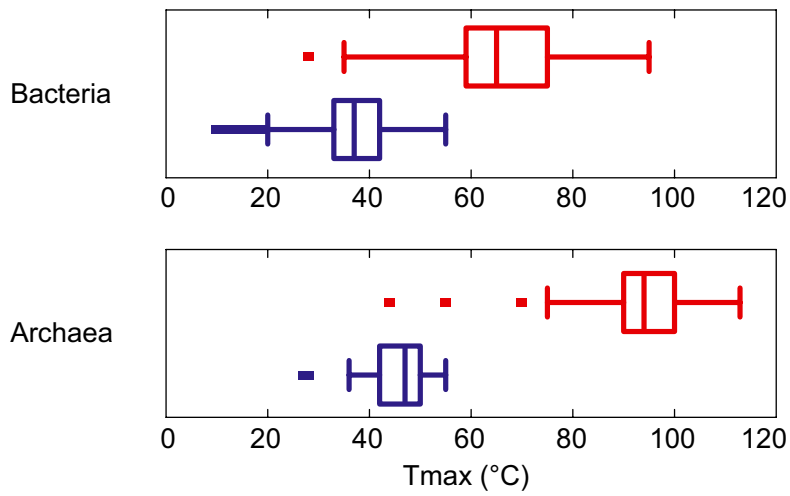

Fig. 3 Distribution of Tmax for positive growth of multi- and unicellular Eukarya, Bacteria, and Archaea sorted according to habitats. (Multicellular Eukarya (Data S1): hydrothermal $N=1$; non-hydrothermal $N=52$; unicellular Eukarya (Data S2): hydrothermal $N=3$; non-hydrothermal $N=162$; Bacteria (Data S3): hydrothermal $N=84$; non-hydrothermal $N=772$; Archaea (Data S4): hydrothermal $N=61$; non-hydrothermal $N=17$ ). Box-and-whisker plots illustrate median values with outliers. 
(a)

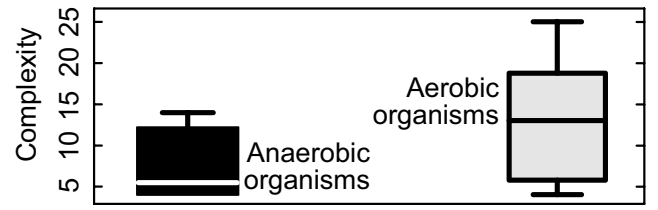

(b)

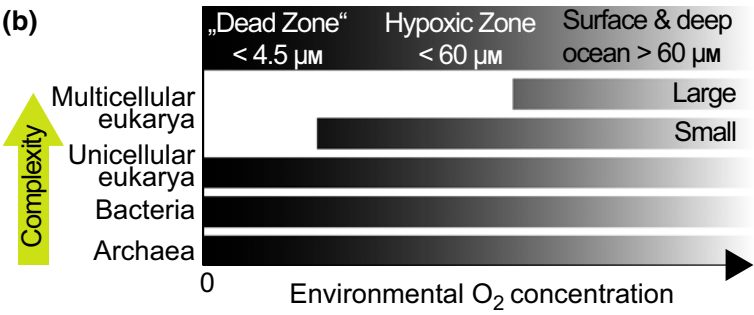

(c) Cooling $\longleftarrow \mathrm{O}_{2}$ crit shift $\longrightarrow$ Warming

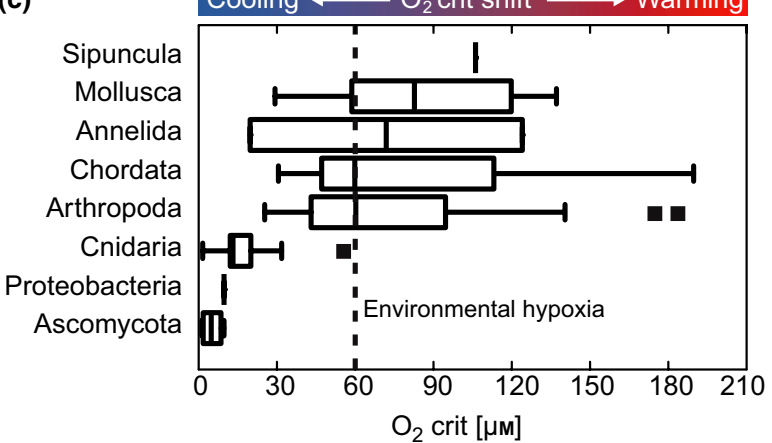

(d)

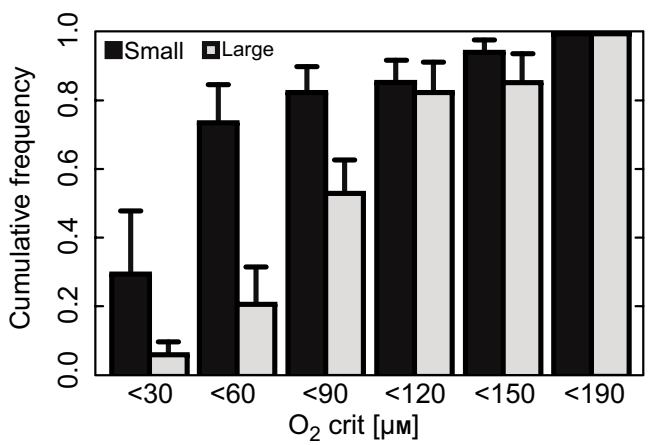

Fig. 4 Oxygen tolerances and organismic complexity across domains. (a) Complexity of anaerobic and aerobic organisms (Table S1). Species from domains and groups, as in Fig. 1 (anaerobic: $N=4$; aerobic: $N=10$ ), shown as box-and-whisker plot. (b) Oxygen ranges (horizontal bars) covered by the respective domains reflect specialization in high ambient oxygen concentrations by uni- and multicellular Eukarya and at large body size. Unicellular organisms from all domains are found under all environmental $\mathrm{O}_{2}$ conditions $(\mathrm{Green}$ arrow indicates increasing organismic complexity from Archaea to multicellular Eukarya). Oxygen concentration thresholds in the ocean are given in the rectangle. (c\&d) Critical oxygen concentrations of marine species, regardless of ambient temperature, (Data S5). (c) Distribution among taxa $(N=74$ in total subdivided into Sipuncula $N=1$, Mollusca $N=10$, Annelida $N=2, \mathrm{Chordata} N=27$, Arthropoda $N=19$, Cnidaria $N=10$, Proteobacteria $N=1$, Ascomycota $N=4$ ), shown as box-and-whisker plots with outliers. The hypoxia threshold characterizing transition to hypoxic communities is inserted as a dashed line. (d) Cumulative histogram of species frequencies (small vs. large, $N=69$, animals only) per defined oxygen concentration ranges. Large multicellular Eukarya are the most hypoxia intolerant, causing the fractional numbers of existing species to decrease with progressive hypoxia and increasing body size. (Error bars are SDs from sampling class boundaries separating small (black) from large (grey) animals).

temperatures (Fig. 3). Their median Tmax was $30{ }^{\circ} \mathrm{C}$ in non-hydrothermal unicellular and $22.5^{\circ} \mathrm{C}$ in non-hydrothermal multicellular Eukarya, significantly lower than in Archaea and Bacteria $(P<0.0001)$. Assuming that Archaea and Bacteria originate from thermophilic ancestors (Di Giulio, 2003), they likely benefit from warming and outcompete the Eukarya at temperatures where these encounter thermal stress.

Field and paleo-observations are in line with our present findings. In the North and South Atlantic, a progressive shift was observed from photoautotrophic systems dominating at high, cooler latitudes to heterotrophic systems prevailing at low, warmer latitudes. This may reflect the heat-induced failure of unicellular eukaryotic algae to compete with Bacteria (Hoppe et al., 2002). However, as net-heterotrophic systems require an import of carbon they rely on the continued presence of photoautotrophic organisms such as cyanobacteria.
In today's warmest oceans, plants, microalgae, and animals thus operate at the limits of their domain, which, being set by complexity, cannot be overcome by acclimatization or evolutionary adaptation. Such heat limits were repeatedly surpassed in earth history, causing e.g., tropical coral communities to retreat from the equator and the lowest latitudes during the last interglacial which was warmer than today (Kiessling et al., 2012). Because organisms specialize in the temperatures of their habitat those in cooler climates display heat limits well below domain-specific thresholds. Upon warming, these limits might then shift during evolutionary adaptation, involving adjustments in protein and membrane structure and functioning (Liberles et al., 2012), until taxon-specific limits are reached. However, the ongoing, large-scale poleward displacements of extant marine animals and plants (Poloczanska et al., 2013) indicate that the rate of climate 
change is presently too fast for significant adaptation to occur, causing species to move and track their preferred temperatures.

Complexity of marine organisms may not only relate to their thermal tolerance but also to their tolerance of low oxygen concentrations (hypoxia), commonly reported at habitat temperatures (Data S1-S5; Fig. 4b). Hypoxia favors species that can either handle reduced oxygen supply, or those that thrive without as facultative and obligate anaerobes. Among the 14 major groups represented in Fig. 1, fully anaerobic organisms cover a narrow range of low complexity levels (Fig. 4a; Table S1). Fully anaerobic Eukarya are mostly unicellular (Dawson \& Pace, 2002); only a single study describes an extremely small multicellular species with a body length of $<0.3 \mathrm{~mm}$ that conquered anoxic habitats (Danovaro et al., 2010). Expanding midwater oxygen minimum zones and an increasing number of oxygen-depleted 'Dead' Zones in warming oceans create favorable conditions for hypoxia tolerant and anaerobic Archaea, Bacteria, and a reduced diversity of unicellular Eukarya (Gooday et al., 2000; Levin, 2010) but exclude the larger animals and plants. Low complexity levels in Archaea, Bacteria, and unicellular Eukarya may enable them to thrive in the range from high environmental oxygen concentrations to anoxic conditions (Fig. 4b), combined with a high capacity of their taxa to exploit anaerobic resources.

Within the Eukarya, the formation of new domains and taxa over evolutionary time, has involved changes in complexity, activity and specialization on higher and narrower oxygen ranges. Interestingly, a burst of evolutionary progress was associated with rising oxygen levels (Lane, 2002; McFall-Ngai et al., 2013) causing a widening from anaerobic to aerobic life options, followed by the evolution of highly energetic aerobic life forms (Bambach et al., 2002). $\mathrm{O}_{2}$ availability also led to an increase in metabolic network complexity, beyond that reached by any anoxic network (Raymond \& Segrè, 2006), partly due to the distribution of pathways across various compartments including mitochondria and chloroplasts in Eukarya. While some unicellular Eukarya live under permanently anoxic conditions, the larger, more complex multicellular Eukarya, i.e. animals and plants, are less tolerant to hypoxia (Díaz \& Rosenberg, 2008) and some depend on oxygen concentrations (Fig. 4c) even higher than the commonly assumed hypoxia threshold of $60 \mu \mathrm{M} \mathrm{O}^{2}$ (Vaquer-Sunyer \& Duarte, 2008).

Hypoxia tolerance is quantified by the critical ambient oxygen concentration $\left(\mathrm{O}_{2 \text { crit }}\right)$ below which resting metabolism falls (Hochachka, 1986) and energy demand is increasingly covered by anaerobic metabolism (Pörtner \& Grieshaber, 1993; Cochran \& Burnett, 1996).
$\mathrm{O}_{2}$ crit values are highly variable among 74 marine species from eight major phyla of animals, fungi, and Bacteria, ranging from $1.23 \mu \mathrm{M} \mathrm{O} \mathrm{O}_{2}$ to $189.70 \mu \mathrm{M} \mathrm{O} \mathrm{O}_{2}$ (Fig. 4c; Data S5). This variability is not apparently influenced by assay temperatures at ambient values and may thus reflect compensation for temperature effects during adaptation of $\mathrm{O}_{2}$ crit to environmental conditions. However, $\mathrm{O}_{2}$ crit values rise with body size (within species) and with complexity levels (within Eukarya and across domains) (Fig. 4d; Carroll, 2001; Lane \& Martin, 2010). Unicellular fungi and Proteobacteria are thus more hypoxia tolerant than the animal phyla. Among animals the cnidarians, one of the most primitive multicellular eukaryan phyla (Cavalier-Smith, 1993), are the most hypoxia tolerant. The more complex arthropods and chordates are the least hypoxia tolerant (Fig. 4b) (Vaquer-Sunyer \& Duarte, 2008).

As a corollary, our results indicate a strong effect of organismic complexity on tolerance to temperature and hypoxia within and across domains. They suggest an explanation of why thermal (and hypoxia) tolerances decrease from Archaea to Bacteria to uni- and then multicellular Eukarya. Organismic complexity likely sets firm limits to evolutionary adaptation of a taxon and cannot be shifted any further. Complex aerobic Eukarya may thus be excluded from ecosystems in the warmest future oceans, as their maximum heat limits are close to today's maximum ocean temperatures at low latitudes. In all oceans, ecosystem functioning involves the participation of organisms from different domains in predator prey and competitive interactions. Our analysis may also form the basis of an understanding of the dynamics of species interactions within and across all domains, and in various climate zones, building on differential performances and tolerances (cf. Pörtner \& Farrell, 2008 for animals only). In the future, there is a need to explore the mechanisms setting and limiting species-specific tolerances and performances in each of the various taxa. The exploration of these mechanistic settings should include the various levels of biological organization from whole organism to molecular, including protein and membrane evolution and biophysics (Liberles et al., 2012). The principle understanding that limits are always set at the highest complexity levels (such as OCLTT in animals) should be suitable to guide such investigations. This not only relates to temperature limits but also to limits of hypoxia tolerance, and is likely involved in interactions of multiple drivers in the oceans (Boyd \& Hutchins, 2012).

\section{Acknowledgements}

Parts of this study have been inspired by discussions during preparation of the IPCC WGII Fifth Accessment Report, chapter 
6 "Ocean Systems" (2014). This study was supported by the PACES program of the AWI, grant no. STO 857/2-1 of the Deutsche Forschungsgemeinschaft (DFG) to DS and grant no. 01 LG $1005 \mathrm{~F}$ of the Federal Ministry of Education and Research (BMBF) to HOP. Thanks to J. Wiest who helped with the set up of the databases. Supporting information includes one pdf file (with one figure and R scripts), one table, and five data files and can be found in the online version of the article. The authors declare no competing financial interests. Readers are welcome to comment on the online version of the paper. Correspondence and requests for materials should be addressed to D.S. (Daniela. Storch@awi.de) or H.O.P. (Hans.Poertner@awi.de).

\section{References}

Bambach RK, Knoll AH, Sepkoski JJ (2002) Anatomical and ecological constraints on Phanerozoic animal diversity in the marine realm. Proceedings of the National Academy of Sciences of the United States of America, 99, 6854-6859.

Benjamini Y, Hochberg Y (1995) Controlling the false discovery rate: a practical and powerful approach to multiple testing. Journal of the Royal Statistical Society Series B-Methodological, 57, 289.

Bopp L, Resplandy L, Orr JC et al. (2013) Mutiple stressors of ocean ecosystems in the 21st century; projections with CMIP5 models. Biogeosciences, 10, 6225-6245.

Boyd PW, Hutchins DA (2012) Understanding the responses of ocean biota to a complex matrix of cumulative anthropogenic change. Marine Ecology Progress Series, 470, 125-135.

Carroll SB (2001) Chance and necessity: the evolution of morphological complexity and diversity. Nature, 409, 1102-1109.

Cavalier-Smith T (1993) Kingdom protozoa and its 18 phyla. Microbiological Reviews, 57, 953-994.

Cochran RE, Burnett LE (1996) Respiratory responses of the salt marsh animals, Fundulus heteroclitus, Leiostomus xanthurus, and Palaemonetes pugio to environmental hypoxia and hypercapnia and to the organophosphate pesticide, azinphosmethyl. Journal of Experimental Marine Biology and Ecology, 195, 125-144.

Copper P (1994) Ancient reef ecosystem expansion and collapse. Coral Reefs, 13, 3-11.

Corkrey R, Olley J, Ratkowsky D, McMeekin T, Ross T (2012) Universality of thermodynamic constants governing biological growth rates. PLOS ONE, 7, e32003.

Costello MJ, Bouchet P, Boxshall G et al. (2013) Global coordination and standardisation in marine biodiversity through the world register of marine species (WoRMS) and related databases. PLOS ONE, 8, e51629.

Daniel RM, van Eckert R, Holden JF, Truter J, Cowan DA (2004) The stability of biomolecules and the implications for life at high temperatures. In: The Subseafloor Biosphere at Mid-Ocean Ridges (eds Wilcock WSD, DeLong EF, Kelley DS, Baross JA, Cary SC), pp. 25-39. AGU, Washington.

Danovaro R, Dell'Anno A, Pusceddu A, Gambi C, Heiner I, Kristensen RM (2010) The first metazoa living in permanently anoxic conditions. BMC Biology, 8, 30

Dawson SC, Pace NR (2002) Novel kingdom-level eukaryotic diversity in anoxic environments. Proceedings of the National Academy of Sciences of the United States of America, 99, 8324-8329.

Deutsch CA, Tewksbury JJ, Huey RB, Sheldon KS, Ghalambor CK, Haak DC, Martin PR (2008) Impacts of climate warming on terrestrial ectotherms across latitude. Proceedings of the National Academy of Sciences of the United States of America, 105, 6668-6672.

Deutsch C, Brix H, Ito T, Frenzel H, Thompson L (2011) Climate-forced variability of ocean hypoxia. Science, 333, 336-339.

Di Giulio M (2003) The universal ancestor was a thermophile or a hyperthermophile: tests and further evidence. Journal of Theoretical Biology, 221, 425-436.

Díaz RJ, Rosenberg R (2008) Spreading dead zones and consequences for marine ecosystems. Science, 321, 926-929.

Fiala-Médioni A (1979) Effects of oxygen tension on pumping, filtration and oxygen uptake in the ascidian Phallusia mammillata. Marine Ecology Progress Series, 1, 49-53. Gilmour AE (1979) Ross ice shelf sea temperatures. Science, 203, 438-439.

Gooday AJ, Bernhard JM, Levin LA, Suhr SB (2000) Foraminifera in the Arabian Sea oxygen minimum zone and other oxygen-deficient settings: taxonomic composition, diversity, and relation to metazoan faunas. Deep-Sea Research Part II-Topical Studies in Oceanography, 47, 25-54.

Haase KM, Petersen S, Koschinsky A et al. (2007) Young volcanism and related hydrothermal activity at $5^{\circ} \mathrm{S}$ on the slow-spreading southern Mid-Atlantic Ridge. Geochemistry, Geophysics, Geosystems, 8, Q11002.
Hochachka PW (1986) Defense strategies against hypoxia and hypothermia. Science, 231, 234-241.

Hoegh-Guldberg O, Bruno JF (2010) The impact of climate change on the world's marine ecosystems. Science, 328, 1523-1528.

Hoppe H-G, Gocke K, Koppe R, Begler C (2002) Bacterial growth and primary production along a north-south transect of the Atlantic Ocean. Nature, 416, 168-171.

Irwin S, Davenport J (2002) Hyperoxic boundary layers inhabited by the epiphytic meiofauna of Fucus serratus. Marine Ecology Progress Series, 244, 73-79.

Karl DM, Bidigare RR, Letelier RM (2001) Long-term changes in plankton community structure and productivity in the North Pacific Subtropical Gyre: the domain shift hypothesis. Deep-Sea Research II, 48, 1449-1470.

Keeling RF, Körtzinger A, Gruber N (2010) Ocean deoxygenation in a warming world. Annual Review of Marine Science, 2, 199-229.

Kiessling W, Simpson C, Beck B, Mewis H, Pandolfi JM (2012) Equatorial decline of reef corals during the last Pleistocene interglacial. Proceedings of the National Academy of Sciences of the United States of America, 109, 21378-21383.

Lane N (2002) Silence of the Aeons, three billion years of microbial evolution. In: Oxygen: The Molecule that Made the World (ed Lane N), pp. 29-53. Oxford University Press, Oxford, UK.

Lane N, Martin W (2010) The energetics of genome complexity. Nature, 467, 929-934.

Levin LA (2010) Anaerobic metazoans: no longer an oxymoron. BMC Biology, 8, 31.

Liberles DA, Teichmann SA, Bahar I et al. (2012) The interface of protein structure, protein biophysics, and molecular evolution. Protein Science, 21, 769-785.

Mantyla AW, Reid JL (1983) Abyssal characteristics of the World Ocean waters. DeepSea Research, 30, 805-833.

McFall-Ngai M, Hadfield MG, Bosch TCG et al. (2013) Animals in a bacterial world, a new imperative for the life sciences. Proceedings of the National Academy of Sciences of the United States of America, 110, 3229-3236.

McKenzie LJ (1994) Seasonal changes in biomass and shoot characteristics of a Zostera capricorni Aschers. Dominant meadow in Cairns Harbour, Northern Queensland. Australian Journal of Marine and Freshwater Research, 45, 1337-1352.

McKenzie LJ, Campbell SJ (2004) Surviving the summer heat: seagrass and corals. Seagrass-Watch News, 19, 1.

Meinshausen M, Smith SJ, Calvin K et al. (2011) The RCP greenhouse gas concentrations and their extensions from 1765 to 2300 . Climatic Change, 109, 213-241.

Moss R, Babiker M, Brinkman S et al. (2008) Towards new scenarios for analysis of emissions, climate change, impacts, and response strategies. Technical Summary. Geneva, Intergovernmental Panel on Climate Change: 25.

Naylor JK, Taylor EW, Bennett DB (1999) Oxygen uptake of developing eggs of Cancer pagurus (Crustacea: Decapoda: Cancridae) and consequent behaviour of the ovigerous females. Journal of the Marine Biological Association of the UK, 79, 305-315.

Parmesan C, Matthews J (2005) Biological impacts of climate change. In: Principles of Conservation Biology (eds Groom MJ, Meffe GK, Carroll CR), pp. 333-374. Sinauer, Sunderland, MA.

Poloczanska ES, Brown CJ, Sydeman WJ et al. (2013) Global imprint of climate change on marine life. Nature Climate Change, 3, 919-925.

Pörtner H-O (2002) Climate variations and the physiological basis of temperature dependent biogeography: systemic to molecular hierarchy of thermal tolerance in animals. Comparative Biochemistry and Physiology A: Molecular and Integrative Physiology, 132, 739-761.

Pörtner H-O, Farrell AP (2008) Ecology: physiology and climate change. Science, 322, 690-692.

Pörtner H-O, Grieshaber MK (1993) Critical $\mathrm{P}_{\mathrm{O} 2}$ in oxyconforming and oxyregulating animals: gas exchange, metabolic rate and the mode of energy production. In: The Vertebrate Gas Transport Cascade Adaptations to Environment and Mode of Life (eds Eduardo J, Bicudo PW), pp. 330-357. CRC Press, Boca Raton.

Pörtner H-O, Knust R (2007) Climate change affects marine fishes through the oxygen limitation of thermal tolerance. Science, 315, 95-97.

Raymond J, Segrè D (2006) The effect of oxygen on biochemical networks and the evolution of complex life. Science, 311, 1764-1767.

Storch D, Fernandez M, Navarrete SA, Pörtner H-O (2011) Thermal tolerance of larval stages of the Chilean kelp crab Taliepus dentatus. Marine Ecology Progress Series, 429, 157-167.

Vaquer-Sunyer R, Duarte CM (2008) Thresholds of hypoxia for marine biodiversity. Proceedings of the National Academy of Sciences of the United States of America, 105, 15452-15457.

Wilcoxon F (1945) Individual comparisons by ranking methods. Biometrics Bulletin, 1, 80-83.

Woese CR, Kandler O, Wheelis ML (1990) Towards a natural system of organisms: proposal for the domains Archaea, Bacteria, and Eucarya. Proceedings of the National Academy of Sciences of the United States of America, 87, 4576-4579. 


\section{Supporting Information}

Additional Supporting Information may be found in the online version of this article:

Figure S1. Oxygen solubility in air-saturated water of different salinities. The black dotted line indicates the transition to hypoxiaadapted communities found below oxygen concentrations of $\approx 60 \mu \mathrm{M} \mathrm{O} \mathrm{O}_{2}$ or $<2 \mathrm{mg} \mathrm{O} \mathrm{O}_{2} \mathrm{l}^{-1}$. Black dotted arrows exemplify the decrease in oxygen solubility in 0 and $40{ }^{\circ} \mathrm{C}$ seawater (35 PSU) upon warming according to the RCP scenario 8.5 predicted for 2100. Table S1. This excel file contains the source data for Figs 1 and 4a. It includes all maximal temperatures of positive growth (Tmax) reported for marine species from different groups of the three domains and their corresponding complexity levels. A second spreadsheet contains a 'key of column B' and a third spreadsheet contains the full references cited in the first spreadsheet 'complexity'.

Data S1. This excel file includes all source data on multicellular eukaryan species and their taxonomic classification, oxygen requirements and assay temperature Tmin and Tmax for growth, habitat preference, and the corresponding reference.

Data S2. This excel file includes all source data on unicellular eukaryan species and their taxonomic classification, oxygen requirements and assay temperature Tmin and Tmax for growth, habitat preference, and the corresponding reference.

Data S3. This excel file includes all source data on species of Bacteria and their taxonomic classification, oxygen requirements and assay temperature Tmin and Tmax for growth, habitat preference, and the corresponding reference.

Data S4. This excel file includes all source data on Archaean species and their taxonomic classification, oxygen requirements and assay temperature Tmin and Tmax for growth, habitat preference, and the corresponding reference.

Data S5. This excel file includes all source data on critical oxygen concentrations of species including their taxonomic classification, weight and the corresponding reference. Furthermore, it includes a second spreadsheet with a 'key of column headings'.

Data S6. R Scripts. 